\title{
As interferências e as limitações metodológicas na dosagem da hemoglobina glicada (A1C)
}

Interferences and methodological limitations with glycated hemoglobin measurements (A1C)

Nairo M. Sumita

A hemoglobina glicada (A1C) tornou-se um exame essencial no controle do paciente diabético após a publicação dos estudos clínicos do Diabetes Control and Complications Trial (DCCT), em 1993(3), e do United Kingdom Prospective Diabetes Study (UKPDS), em 1998 ${ }^{(9)}$. Esses estudos revelaram que a manutenção do nível de A1C abaixo de 7\%, no paciente diabético, proporcionaria redução considerável no risco para o desenvolvimento de complicações crônicas do diabetes mellitus ${ }^{(3,9)}$.

No ano de 2009, uma comissão internacional de especialistas definiu o uso da A1C também para fins diagnósticos, estabelecendo o valor acima de 6,5\%, confirmado em uma segunda dosagem, como critério diagnóstico para o diabetes mellitus. Para essa finalidade, a metodologia para dosagem da A1C deve possuir a certificação do National Glycohemoglobin Standardization Program (NGSP)(1).

O NGSP avalia os métodos existentes no mercado mundial para dosagem de A1C com o intuito de verificar se eles produzem resultados compatíveis com a metodologia utilizada pelo DCCT. Caso essa equivalência não seja observada, avalia-se a possibilidade de se estabelecer um cálculo matemático para correção do resultado, tornando o método rastreável em relação àquele utilizado pelo $\mathrm{DCCT}^{(4-8)}$.

O conhecimento prévio dos interferentes é fundamental para que o laboratório clínico assessore o médico diante de um resultado aparentemente inconsistente. Nesse sentido, o NGSP descreve os potenciais interferentes com a finalidade de auxiliar os laboratórios clínicos na escolha da melhor metodologia para a dosagem da A1C, a qual está disponível no site www.ngsp.org/interf.asp ${ }^{(4)}$.

Entre os principais interferentes, destacam-se:

- presença das variantes genéticas da hemoglobina - as hemoglobinas $S$ ou $C$, por exemplo, na condição heterozigótica, podem produzir interferências na medida da $\mathrm{A} 1 \mathrm{C}$, resultando valores falsamente elevados ou diminuídos, de acordo com o tipo de método de ensaio utilizado. A dosagem de A1C não se aplica nas condições de homozigose para hemoglobinas anômalas, por qualquer metodologia, pois, nesses casos, a hemoglobina A encontra-se praticamente ausente. Nessas situações, um exame alternativo, como a frutosamina ou albumina glicada, poderá ser útil $(2,5,6,8)$;

- as doenças que alteram o tempo de sobrevida das hemácias, como anemia hemolítica e hemorragia, podem resultar valores falsamente baixos ${ }^{(5,6,8)}$;

- a presença de grandes quantidades de vitaminas C e E é descrita como um dos fatores que podem induzir a resultados falsamente diminuídos por inibirem o processo de glicação da hemoglobina $^{(5,6,8)}$;

Patologista clínico; doutor pela Faculdade de Medicina da Universidade de São Paulo (FMUSP); professor assistente da disciplina de Patologia Clínica da FMUSP; diretor do Serviço de Bioquímica Clínica da Divisão do Laboratório Central do Hospital das Clínicas da FMUSP; assessor médico em Bioquímica Clínica - Fleury Medicina e Saúde; diretor cientíico da Sociedade Brasileira de Patologia Clínica/Medicina Laboratorial (SBPC/ML). 
- nos estados de anemia por carência de ferro, vitamina B12 ou folato, nos quais ocorre aumento da sobrevida das hemácias, pode ser observada falsa elevação dos valores de $\mathrm{A} 1 \mathrm{C}^{(5,6,8)}$;

- a presença de hemoglobinas quimicamente modificadas, como, por exemplo, a hemoglobina carbamilada associada à uremia e a hemoglobina acetilada formada após a ingestão de elevadas doses de salicilatos, pode elevar falsamente os resultados ${ }^{(5,6,8)}$;

- hipertrigliceridemia, hiperbilirrubinemia, alcoolismo crônico e uso crônico de opiáceos são outras condições clínicas que podem elevar o resultado da $\mathrm{A} 1 \mathrm{C}^{(5,6,8)}$;

- a pré-A1C ou a base de Schiff, fração lábil da A1C, pode ser importante interferente na dosagem, ainda que as metodologias mais modernas permitam a remoção desse interferente ${ }^{(5,6,8)}$.

Um artigo publicado nesta edição do Jornal Brasileiro de Patologia Clínica/Medicina Laboratorial traz uma contribuição muito importante no estudo da interferência das variantes da hemoglobina na dosagem da A1C em pacientes diabéticos na cidade de Salvador-BA, onde a prevalência de portadores de variantes de hemoglobina $\mathrm{S}$ e $\mathrm{C}$ é relativamente elevada. Além disso, destaca a necessidade de alertar a comunidade médica no que tange à abordagem adequada no uso desse parâmetro laboratorial, tanto no diagnóstico como no acompanhamento do diabetes mellitus.

\section{Referências}

1. AMERICAN DIABETES ASSOCIATION. Standards of medical care in diabetes 2012. Diabetes Care, v. 35, Suppl. 1, p. S11-63, 2012.

2. ANDRIOLO, A.; VIEIRA, J. G. H. Diagnóstico e acompanhamento laboratorial do diabetes mellitus. In: ANDRIOLO. A. Guias de medicina ambulatorial e hospitalar UNIFESP/Escola Paulista de Medicina. Medicina Laboratorial. 2. ed. São Paulo: Manole, 2008. p. 37-42.

3. DCCT RESEARCH GROUP. Diabetes Control and Complications Trial (DCCT). The effect of intensive treatment of intensive treatment of diabetes on the development and progression of long-term complications in insulin-dependent diabetes mellitus. N Engl J Med, v. 329, p. 977-86, 1993.

4. National Glycohemoglobin Standardization Program - NGSP. HbA1c Assay Interferences. HbA1c methods: effects of variants ( $H b C, H b S, H b E$ and $H b D$ traits) and elevated fetal hemoglobin (HbF). Disponível em: http://www.ngsp. org/interf.asp. Acesso em: out. 2012.

5. PIMAZONI NETTO, A. et al. Atualização sobre hemoglobina glicada (HbA1C) para avaliação do controle glicêmico e para o diagnóstico do diabetes: aspectos clínicos e laboratoriais. J Bras Patol, v. 45, n. 1, p. 31-48, 2009.

6. SACKS, D. B. Diabetes mellitus. In: BURTIS, C. A.; ASHWOOD, E. R.; BRUNS, D. E. Tietz textbook of clinical chemistry and molecular diagnostics. 5. ed. St. Louis: Elsevier Saunders, 2012. p. 1415-56.

7. SUMITA, N. M.; ANDRIOLO, A. Importância da determinação da hemoglobina glicada no monitoramento do paciente portador de diabetes mellitus. J Bras Patol, v. 42, n. 3, editorial, 2006.

8. SUMITA, N. M.; ANDRIOLO, A. Importância da hemoglobina glicada no controle do diabetes mellitus e na avaliação de risco das complicações crônicas. J Bras Patol, v. 44, n. 3, p. 169-74, 2008.

9. UK PROSPECTIVE DIABETES STUDY GROUP. Intensive blood glucose control with sulphonylureas or insulin compared with conventional treatment and risk of complications in patients with type 2 diabetes. Lancet, v. 352, p. 837-53, 1998. 